\title{
Nitric oxide, 8-hydroxydeoxyguanosine, and total antioxidant capacity in human seminal plasma of infertile men and their relationship with sperm parameters
}

\author{
Maryam Gholinezhad' ${ }^{1}$ Azadeh Aliarab², Ghasem Abbaszadeh-Goudarzi ${ }^{3}$, Yousefreza Yousefnia-Pasha', Niusha Samadaian ${ }^{4}$, \\ Korush Rasolpour-Roshan ${ }^{5}$, Hemat Aghagolzadeh-Haji ${ }^{5}$, Milad Mohammadoo-Khorasani ${ }^{6}$ \\ 'Infertility and Reproductive Health Research Center, Health Research Institute, Babol University of Medical Sciences, Babol; ${ }^{2}$ Department of Clinical \\ Biochemistry, School of Medicine, Shahid Beheshti University of Medical Sciences, Tehran; ${ }^{3}$ Department of Medical Biotechnology, School of Medicine, \\ Shahroud University of Medical Sciences, Shahroud; ${ }^{4}$ Department of Medical Genetics, School of Medicine, Tehran University of Medical Sciences, \\ Tehran; ${ }^{5}$ Cellular and Molecular Biology Research Center, Babol University of Medical Sciences, Babol; ${ }^{6}$ Department of Clinical Biochemistry, Faculty of \\ Medicine, Gonabad University of Medical Sciences, Gonabad, Iran
}

Objective: Oxidative stress plays a key role in the pathogenesis of male infertility. But, the adverse effects of oxidative biomarkers on sperm quality remain unclear. This study aimed to investigate the levels of nitric oxide (NO), 8-hydroxydesoxyguanosine (8-OHdG), and total antioxidant capacity (TAC) oxidative biomarkers in seminal plasma and their relationship with sperm parameters.

Methods: A total of 77 volunteers participated in the study, including fertile $(n=40)$ and infertile men $(n=37)$. NO, 8-OHdG, and TAC levels were measured using the ferric reducing ability of plasma, Griess reagent method and an enzyme-linked immunosorbent assay kit, respectively. Results: The mean values of sperm parameters in the infertile group were significantly lower than those in the fertile group $(p<0.001)$. The mean 8-OHdG in the seminal plasma of infertile men was significantly higher $(p=0.013)$ than those of controls, while the mean TAC was signifcantly lower $(p=0.046)$. There was no significant difference in NO level between the two groups. The elevated seminal 8-OHdG levels were negatively correlated with semen volume, total sperm counts and morphology $(p<0.001, p=0.001$ and $p=0.052$, respectively). NO levels were negatively correlated with semen volume, total sperm counts and morphology $(p=0.014, p=0.020$ and $p=0.060$, respectively). Positive correlations between TAC and both sperm count and morphology ( $p=0.043$ and $p=0.025$, respectively) were also found.

Conclusion: These results suggested that increased levels of NO and 8-OHdG in seminal plasma could have a negative effect on sperm function by inducing damage to the sperm DNA hence their fertility potentials. Therefore, these biomarkers can be useful in the diagnosis and treatment of male infertility.

Keywords: 8-Hydroxydeoxyguanosine; Infertile men; Nitric oxide; Oxidative stress; Semen; Total antioxidant capacity

Received: July 12, 2019 . Revised: September 10, 2019 · Accepted: September 23, 2019 Corresponding author:Yousefreza Yousefnia-Pasha Infertility and Reproductive Health Research Center, Health Research Institute, Babol University of Medical Sciences, Ganj Afrooz Ave, Babol 4717647745, Iran Tel: +98-11-32274881-2 Fax:+98-11-32274880 E-mail:zeinalmahtab@yahoo.com Co- Corresponding author: Maryam Gholinezhad

Infertility and Reproductive Health Research Center, Health Research Institute, Babol University of Medical Sciences, Ganj Afrooz Ave, Babol 4717647745, Iran Tel:+98-11-32274881-2 Fax:+98-11-32274880 E-mail:m.gholinezhadchari@gmail.com

*Part of the work (samples collection and semen analysis) was presented from the IVF Center in Fatemeh Zahra Hospital in Babol, Iran.

This is an Open Access article distributed under the terms of the Creative Commons Attribution Non-Commercial License (https://creativecommons.org/licenses/by-nc/4.0/) which permits unrestricted non-commercial use, distribution, and reproduction in any medium, provided the original work is properly cited.

\section{Introduction}

Oxidative stress (OS) is now considered as one of the important causes of idiopathic male infertility [1,2]. It is associated with increased cellular damages caused by oxygen-derived free radicals, or byproducts from the metabolism of oxygen metabolites, namely reactive oxygen species (ROS) [1,2]. Leukocytes and immature, or abnormal spermatozoa are the major endogenous sources of ROS in the seminal plasma [3,4]. Although limited levels of ROS are necessary for the processes of spermatozoa maturation, capacitation, hy- 
peractivation, acrosome reaction, and sperm-oocyte fusion, increased content of ROS can attack sperm DNA, lipids and proteins, which is subsequently associated with cell death, poor quality of sperm, and male infertility $[4,5]$. The excessive levels of ROS are commonly overwhelmed by the body's antioxidant defense mechanisms; total antioxidant capacity (TAC) that contains both enzymatic and non-enzymatic antioxidant molecules such as superoxide dismutase (SOD), catalase, glutathione peroxidase, ascorbate, vitamin $\mathrm{E}$, pyruvate, glutathione. However, spermatozoa are unable to restore the oxidative damage due to (1) the high content of polyunsaturated fatty acids in their cell membrane which makes them highly susceptible to ROS by inducing lipid peroxidation, (2) the partial loss of antioxidant protection due to the limit volume of cytoplasm, (3) their lack of the necessary cytoplasmic enzyme repair systems [1-5].

Nitric oxide (NO) is an oxygen free radical molecule that plays a key role in the regulation of numerous physiological processes such as sperm hyperactivation, acrosome reaction, capacitation, and sperm fertilization [6]. It is synthesized under the enzymatic activity of NO synthase (NOS) from L-arginine and L-citrulline [7]. Although NO has a physiological function at low concentration, it causes pathological damages to cells at high concentration. The cytotoxic effects of NO are mediated indirectly through its interaction with the superoxide anion $\left(\mathrm{O}_{2}^{-}\right)$yielding of proxy nitrite anion ( $\left.\mathrm{ONOO}^{-}\right)$and proxy nitrous acid $(\mathrm{ONOOH})$. These activate molecules are strong oxidant that can cause molecular damages or apoptosis to a variety of tissues [8]. However, the effects of NO on sperm function are dose-dependent. At physiological conditions, low NO level plays an essential role in sperm function such as sperm motility [9], capacitation, acrosome reaction [10], whereas an increased level of NO can be associated with a decrease in human sperm motility and viability [11]. Recently, different studies show that expression of NOS regulates normal spermatogenesis in the testis, and the NOS gene variants (G894T, T-786C, and $4 \mathrm{a} 4 \mathrm{~b}$ ) are potentially involved in the impairment of spermatogenesis and sperm function $[12,13]$. On the other hand, it has also been established that high NO levels have harmful effects on DNA [14]. The integrity of sperm DNA is an important factor in successful fertility and embryo development. High levels of ROS are associated with oxidative DNA damages as revealed by an accumulation of the oxidized guanine residue 8-hydroxydesoxyguanosine (8-OHdG), a well-known biomarker of DNA oxidation [15].

There is no report on the relation between NO level and oxidative DNA damage (8-OHdG) in seminal plasma. The aim of the present study is to determine the concentration of $\mathrm{NO}, 8-\mathrm{OHdG}$, and TAC in the seminal plasma and their correlations with semen parameters (volume, concentration, motility, and morphology) in the fertile and infertile men.

\section{Methods}

\section{Subjects}

In this case-control study, a total of 77 semen samples were provided from individuals with the age ranging between 24 to 35 years old, who referred to the in vitro fertilization (IVF) center in Fatemeh Zahra Hospital, Babol, Iran, 2018. Before samples collection, a questionnaire on age and lifestyle also was provided. The subjects were classified into two groups; control group including 40 fertile and healthy men with a normal semen analysis (normozoospermic) and 37 infertile patients with a history of primary infertility for at least 1 year and an abnormal semen analysis. The Ethics Committee of Babol University of Medical Sciences approved the study protocol. The written informed consent was obtained from all individual participants included in the study. Individuals were excluded from the study with a significant medical history or signs of defective androgenization or testicular trauma, cryptorchidism, vasectomy, endocrine disorders, leukocytospermia, sexually transmitted disease, alcohol consumption, and smoking.

\section{Sample collection and semen analysis}

The semen samples were collected into sterile container after a period for $2-3$ days of abstinence at the IVF center of an infertility clinic. Routine semen analysis was performed within 1 hour according to World Health Organization guidelines [16]. Specimens were allowed to liquefy at $37^{\circ} \mathrm{C}$ for 30 minutes, and then sperm concentrations, percentage of motile sperm and sperm with normal morphology objectively were evaluated using microscopic examination. Sperm morphology was evaluated according to the Kruger's criteria, in which sperm morphology $<4 \%$ were considered as abnormal [17]. After semen analysis, samples were centrifuged at $4,000 \mathrm{~g}$ for 10 minutes and stored at $20^{\circ} \mathrm{C}$ for measurement of the OS biomarkers.

\section{8-OHdG measurement in seminal plasma}

The levels of 8-OHdG in $50 \mu \mathrm{L}$ of seminal plasma were measured using an enzyme-linked immunosorbent assay (ELISA kit; CSB-E10140h, 96T, Cusabio, Wuhan, China). The color reaction product was then detected by ELISA reader at $450 \mathrm{~nm}$, and the concentration of 8-OHdG was calculated from a standard curve. The final results were expressed as $\mathrm{ng} / \mathrm{mL}$.

\section{NO measurement in seminal plasma}

The total stable oxidation products of $\mathrm{NO}$ metabolism $\left(\mathrm{NO}_{2}^{-} / \mathrm{NO}_{3}{ }^{-}\right)$ of seminal plasma were assessed using a Griess reagent consisting of sulfanilamide (SULF) and N-(1-Naphthyl) ethylenediamine dihydrochloride (NEDD) [18]. The frozen semen was allowed to thaw until it reached a temperature of $25^{\circ} \mathrm{C}$ that was followed by being deprot- 
einized by zinc sulfate solution (Sigma-Aldrich, St. Louis, MO, USA). The liquefied semen samples were centrifuged at $12,000 \mathrm{~g}$ for 10 minutes at room temperature. Then, $300 \mu \mathrm{L}$ of the clear supernatant was mixed with Griess reagents including $300 \mu \mathrm{L}$ SULF ( $2 \% \mathrm{w} / \mathrm{v})$ in $5 \%$ hydrochloric acid $(\mathrm{HCl})$ and $300 \mu \mathrm{l}$ NEDD $(0.1 \% \mathrm{w} / \mathrm{v}$; Sigma, St. Louis, MO, USA) in $\mathrm{H}_{2} \mathrm{O}$ in a test tube, while for the reduction of nitrate to nitrite, $300 \mu \mathrm{L}$ saturated solutions of vanadium (III) chloride (VCl 3, Sigma) in $1 \mathrm{M} \mathrm{HCl}$ was added and incubated for two hours at $30^{\circ} \mathrm{C}$ in the dark. Then the absorbance of samples was recorded at $540 \mathrm{~nm}$ with a spectrophotometer against a blank containing the same concentrations of ingredients but no biological sample. Linear regression was used to determine NO concentration from the standard curve of $\mathrm{NaNO}_{2}$. The final results were expressed as $\mu \mathrm{mol} / \mathrm{L}$.

\section{TAC measurement in seminal plasma}

The TAC of seminal plasma was evaluated using the ferric reducing ability of plasma (FRAP) method according to the method described by Benzie and Strain [19]. This method measures the ability of antioxidants of a sample to reduce ferric tripyridyltriazine ( $\mathrm{Fe}^{3+}-\mathrm{TPTZ}$ ) to a ferrous form $\left(\mathrm{Fe}^{2+}\right)$. In brief, the working FRAP reagent was prepared by mixing $300 \mathrm{mmol} / \mathrm{L}$ acetate buffer $\mathrm{pH}=(3.1 \mathrm{~g}$ sodium acetate $\times$ $3 \mathrm{H}_{2} \mathrm{O}$ [Merck, Darmstadt, Germany] and $16 \mathrm{~mL}$ acetic acid in 1,000 $\mathrm{mL}$ buffer solution) with $10 \mathrm{mmol} / \mathrm{L}$ 2,4,6,-tripyridyl-s-triazine (TPTZ, Merck), $40 \mathrm{mmol} / \mathrm{L} \mathrm{HCl}$ (Merck), and $20 \mathrm{mmol} / \mathrm{L} \mathrm{FCl}_{3} \times 6 \mathrm{H}_{2} \mathrm{O}$ (Sigma) in a 10:1:1 ratio, just before use. Then, $1.5 \mathrm{~mL}$ of the working FRAP reagent was aliquoted into a glass tube and warmed to $37^{\circ} \mathrm{C}$ for 5 min- utes. Subsequently, $50 \mu \mathrm{L}$ of diluted plasma (total of semen samples centrifuged at $12,000 \mathrm{~g}$ for 7 minutes, and diluted $1: 10 \mathrm{v} / \mathrm{v}$ in distilled water), $50 \mu \mathrm{L}$ of distilled water (reagent-free), as well as $50 \mu \mathrm{L}$ of each of the standard solutions ( $\mathrm{FeSO}_{4} .7 \mathrm{H}_{2} \mathrm{O} ; 1,000,500,250$, and $125 \mu \mathrm{M}$; Sigma) were added to $1.5 \mathrm{~mL}$ FRAP reagent and heated in $37^{\circ} \mathrm{C}$ for 10 minutes. Then, the absorbance of samples was measured at 593 $\mathrm{nm}$ with a spectrophotometer (UV1600). The final results were expressed as $\mu \mathrm{M} / \mathrm{L}$.

\section{Statistical analysis}

Independent $t$-test was used for comparisons to examine differences between the data groups, NO, 8-OHdG, and TAC levels and other parameters including age, volume, sperm count, total sperm counts, percentage of normal morphology and motility of sperm between the two groups; fertile, and infertile men. These data were reported as mean \pm standard deviation. The odds ratios were presented together with their $95 \%$ confidence intervals using SPSS ver. 18.0 (SPSS Inc., Chicago, IL, USA). A linear regression (Spearman) model was applied to the relationship between NO, 8-OHdG, and TAC levels with sperm parameters. In all cases, a $p$-value $\leq 0.05$ was considered as statistical significant.

\section{Results}

1. Characteristics of the study subjects and semen parameters

The mean values of sperm parameters quality in infertile men and

Table 1. Comparison of sperm parameters between two groups of the fertile and infertile men

\begin{tabular}{lcccc}
\hline Variable & Fertile $(\mathrm{n}=40)$ & Infertile $(\mathrm{n}=37)$ & Mean difference $(95 \% \mathrm{Cl})$ & $p$-value $\mathrm{a}^{\mathrm{a}}$ \\
\hline Age $(\mathrm{yr})$ & $30.05 \pm 4.59$ & $30.43 \pm 3.32$ & $-0.38(-2.21$ to 1.45$)$ & 0.67 \\
Volume $(\mathrm{mL})$ & $3.76 \pm 1.21$ & $3.54 \pm 1.51$ & $0.22(-0.40$ to 0.84$)$ & 0.48 \\
Count $\left(\times 10^{6} / \mathrm{mL}\right)$ & $85.00 \pm 20.38$ & $26.05 \pm 26.80$ & $58.94(48.18$ to 69.70$)$ & $<0.001$ \\
Total count $\left(\times 10^{6}\right)$ & $313.12 \pm 129.23$ & $99.60 \pm 115.81$ & $213.51(157.63$ to 269.39$)$ & $<0.001$ \\
Motility $(\%)$ & $60.00 \pm 7.51$ & $38.81 \pm 17.71$ & $21.18(15.09$ to 27.28$)$ & $<0.001$ \\
Morphology $(\%)$ & $19.00 \pm 6.28$ & $6.05 \pm 4.94$ & $12.94(10.36$ to 15.52$)$ & $<0.001$ \\
\hline
\end{tabular}

Values are presented as mean \pm standard deviation.

Fertile, healthy control group with a normal semen analysis; Infertile, patients with primary infertility and abnormal semen analysis; $\mathrm{Cl}$, confidence interval.

a) The $p<0.05$ was considered statistically significant. Independent $t$-test was used for comparisons between two groups.

Table 2. Comparison of 8-OHdG, NO, and TAC concentration in seminal plasma between fertile and infertile groups

\begin{tabular}{lcccc}
\hline Variable & Fertile $(n=40)$ & Infertile $(n=37)$ & Mean difference $(95 \% \mathrm{Cl})$ & $p$-value $\mathrm{a}^{\mathrm{a})}$ \\
\hline 8-OHdG $(\mathrm{ng} / \mathrm{mL})$ & $304.15 \pm 126.29$ & $389.66 \pm 165.63$ & $-85.51(-152.08$ to -18.94$)$ & 0.013 \\
NO $(\mu \mathrm{mol} / \mathrm{L})$ & $6.39 \pm 4.59$ & $7.94 \pm 5.91$ & $-1.54(-3.94$ to 0.84$)$ & 0.202 \\
TAC $(\mu \mathrm{M} / \mathrm{L})$ & $2,015.50 \pm 670.95$ & $1,697.11 \pm 708.18$ & $318.41(5.31$ to 631.52$)$ & 0.046 \\
\hline
\end{tabular}

Values are presented as mean \pm standard deviation.

8-OHdG, 8-hydroxydesoxyguanosine; NO, nitric oxide; TAC, total antioxidant capacity; Fertile, healthy control group with a normal semen analysis; Infertile, patients with primary infertility and abnormal semen analysis; $\mathrm{Cl}$, confidence interval.

a) The $p<0.05$ was considered statistically significant. Independent $t$-test was used for comparisons between two groups. 
the control group are shown in Table 1. The initial study samples consisted of 40 normozoospermic men (51.94\%) and 37 infertile men (48.05\%), of whom group, $27.03 \%$ oligoteratozoospermia, $5.40 \%$ oligozoospermia, $32.43 \%$ oligoasthenoteratozoospermia, $5.40 \%$ asthenozoospermia, $13.51 \%$ asthenoteratozoospermia and $16.21 \%$ were teratozoospermia. There was not a significant difference in mean age, and also volume between two groups. The mean values of sperm counts, total sperm counts, motility and normal morphology of sperm in the infertile group were significantly lower than that in the fertile group $(p<0.001)$.

\section{Mean values of OS biomarkers in seminal plasma of fertile and infertile groups}

The mean values of OS biomarkers in seminal plasma of infertile men and the control group are depicted in Table 2. The mean 8OHdG levels in the seminal plasma of infertile men $(389.66 \pm 165.63$ $\mathrm{ng} / \mathrm{mL}$ ) were significantly higher than that in of the control group (304.15 $\pm 126.29 \mathrm{ng} / \mathrm{mL}, p=0.013$ ), while we didn't find a significant difference in mean of NO levels in the seminal plasma between the two groups $(p=0.202)$. However, a trend for higher means NO value was observed in the seminal plasma of infertile patients. In addition, the mean TAC in the seminal plasma was significantly lower for infertile men than for men in the control group $(1,697.11 \pm 708.18$ and $2,015.50 \pm 670.95 \mu \mathrm{M} / \mathrm{L}, p=0.046)$.

\section{Correlations between OS biomarkers and sperm parameters in fresh semen}

The correlation coefficients of various parameters in all subjects are demonstrated in Table 3 and Figure 1. A positive correlation was observed between semen volume, and total sperm counts $(p<0.001, r=0.437)$, the total sperm counts and sperm motility $(p<0.001, r=0.435)$, the total sperm count and normal sperm morphology $(p<0.001, r=0.638)$, sperm motility, and morphology ( $p<0.001, r=0.576)$. A negative correlation was seen between elevated 8-OHdG levels in the seminal plasma with semen volume $(p<0.001, r=-0.432)$, total sperm counts $(p=0.001$, $r=-0.375)$ and normal sperm morphology $(p=0.052, r=-0.223)$. Also, negative correlations were also observed between NO levels and semen volume ( $p=0.014, r=-0.279)$, total sperm counts $(p=0.020, r=-0.265)$ and normal sperm morphology $(p=0.060, r=-0.216)$. Positive correlations were found between TAC and sperm count $(p=0.043, r=0.232)$ and between TAC and normal sperm morphology ( $p=0.025, r=0.255)$. A positive correlation was observed between elevated $8-\mathrm{OHdG}$ and NO levels in seminal plasma while 8-OHdG levels were negatively correlated with TAC. But, the results not statistically significant (Table 3, Figure 1).

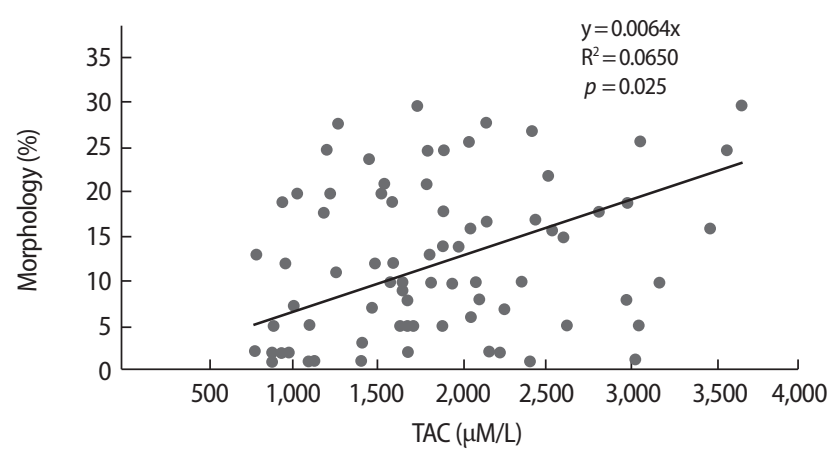

Figure 1. Correlations between total antioxidant capacity (TAC) in seminal plasma and sperm morphology.

Table 3. Pearson correlation analysis between oxidative biomarkers in seminal plasma with sperm parameters

\begin{tabular}{|c|c|c|c|c|c|c|c|c|c|}
\hline Variable & $\begin{array}{l}\text { Semen sample } \\
\quad(n=77)\end{array}$ & 8-OHdG & NO & TAC & Volume & Count & Total count & Motility & Morphology \\
\hline \multirow[t]{2}{*}{ 8-OHdG } & Pearson correlation & 1 & 0.151 & -0.146 & $-0.432^{\mathrm{a})}$ & -0.175 & $-0.375^{\mathrm{a})}$ & -0.140 & -0.223 \\
\hline & Significant (2-tailed) & & 0.190 & 0.206 & 0.000 & 0.127 & 0.001 & 0.223 & 0.052 \\
\hline \multirow[t]{2}{*}{ NO } & Pearson correlation & & 1 & -0.045 & $-0.279^{b)}$ & -0.166 & $-0.265^{b)}$ & -0.143 & -0.216 \\
\hline & Significant (2-tailed) & & & 0.695 & 0.014 & 0.149 & 0.020 & 0.214 & 0.060 \\
\hline \multirow[t]{2}{*}{$\mathrm{TAC}$} & Pearson correlation & & & 1 & 0.021 & $0.232^{b)}$ & 0.115 & 0.114 & $0.255^{b)}$ \\
\hline & Significant (2-tailed) & & & & 0.855 & 0.043 & 0.318 & 0.325 & 0.025 \\
\hline \multirow[t]{2}{*}{ Volume } & Pearson correlation & & & & 1 & 0.036 & $0.437^{\mathrm{a})}$ & -0.017 & 0.168 \\
\hline & Significant (2-tailed) & & & & & 0.759 & 0.000 & 0.886 & 0.144 \\
\hline \multirow[t]{2}{*}{ Sperm count } & Pearson correlation & & & & & 1 & $0.825^{a)}$ & $0.607^{\mathrm{a})}$ & $0.722^{\mathrm{a})}$ \\
\hline & Significant (2-tailed) & & & & & & 0.000 & 0.000 & 0.000 \\
\hline \multirow[t]{2}{*}{ Total sperm count } & Pearson correlation & & & & & & 1 & $0.435^{\mathrm{a})}$ & $0.638^{\mathrm{a})}$ \\
\hline & Significant (2-tailed) & & & & & & & 0.000 & 0.000 \\
\hline \multirow[t]{2}{*}{ Motility } & Pearson correlation & & & & & & & 1 & $0.576^{\mathrm{a})}$ \\
\hline & Significant (2-tailed) & & & & & & & & 0.000 \\
\hline
\end{tabular}

8-OHdG, 8-hydroxydesoxyguanosine; NO, nitric oxide; TAC, total antioxidant capacity.

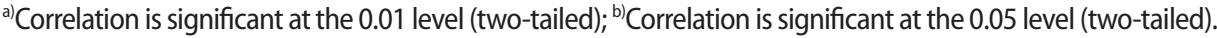




\section{Discussion}

In a healthy body, there is a balance between pro-oxidants and antioxidants in the biological system. However, under pathological conditions, the uncontrolled production of ROS exceeds the antioxidants capacity of the seminal plasma, resulting in oxidative damage [1-4]. In this study, we investigated the relationship between OS biomarkers including NO, 8-OHdG, and TAC levels of seminal plasma with low sperm parameters to show an increase in infertility rate in men.

In the present study, a trend for higher mean NO value was observed in seminal plasma of infertile patients compared with fertile men. But, this difference was not statistically significant between two groups. In contrast, several studies provided evidence that NO concentration in seminal plasma of infertile men is significantly higher than those in normozoospermic fertile group $[7,20]$. In another study, NO concentrations were significantly higher in oligo and asthenozoospermic patients with and without varicocele than in healthy control men [21].

Several studies have shown bilateral effects of NO on sperm parameters $[11,20,22]$. In our study, there were negative correlations between NO level of seminal plasma with semen volume, total sperm counts, and sperm morphology. In agreement with some reports $[22,23]$, in the present study there was no significant difference in NO concentration between the fertile, and infertile subjects and also there was no correlation between NO level and sperm motility. On the other hand, a significant negative correlation was reported between the high NO level in seminal plasma with morphology, sperm count [11] viability [20] and sperm motility [7,9] in infertile men, whereas lower NO levels under physiological conditions have been shown to increase sperm motility [24]. On the contrary, it has been reported that the concentration of NO in seminal plasma does not correlate with sperm motility and sperm concentration [22,23]. Huang et al. [22] observed a significant positive correlation between seminal plasma NO levels and sperm morphology. They suggested that decreased NO levels are associated with defects in sperm morphology. Moreover, in another study by Revelli et al. [23], they demonstrated no correlation between NO concentration and semen parameters. Overall, these findings suggest that $\mathrm{NO}$ has a bilateral role under in pathological and physiological conditions process, being both a cytotoxic and necessary molecule for normal sperm function and particularly sperm motility depends on the alternative redox state and relative NO level [25]. The primary mechanism of high NO level that leads to the reduction of sperm motility is likely to be inhibition of mitochondrial respiration and DNA synthesis by Nitrosylation and resulting in a depletion of adenosine triphosphate in the cell [25]. As a result, the dual effects of NO depend on its concentration and reactions with $\mathrm{ROS}$ such as $\mathrm{O}_{2}{ }^{-}$, and $\mathrm{H}_{2} \mathrm{O}_{2}$ [25]. $\mathrm{NO}$ may react rapidly with $\mathrm{O}_{2}^{-}$to form highly toxic, proxy nitrite $\left(\mathrm{ONOO}^{-}\right)$that is a powerful radical [26], so $\mathrm{ONOO}^{-}$can adversely interact with most biomolecules and leading to oxidation of lipid, protein, and DNA, activation of matrix metalloproteinases and inactivation of several enzymes, particularly mitochondrial enzymes, and also can induce cell death by apoptosis or necrosis [8]. Moreover, $\mathrm{NO}$ and $\mathrm{NOO}_{2}^{-}$can directly induce DNA damage. Sheikh et al. [27] reported a significant positive correlation between $\mathrm{NO}$ levels in seminal plasma and sperm DNA fragmentation.

Several studies have shown that high level of 8-OHdG, one of the best marker of DNA oxidation, in sperm is closely associated with semen quality parameters and male infertility $[15,28]$. The results of this study showed that 8-OHdG levels in seminal plasma were significantly correlated with semen volume, total sperm counts, and normal sperm morphology. In agreement with our results, the mean values of 8-OHdG level were also significantly higher in infertile subjects than healthy fertile men $[28,29]$. In another study, infertile men with varicocele exhibited higher $8-\mathrm{OHdG}$ levels in seminal plasma and spermatozoa than the subjects without varicocele. Moreover, there were negative correlations between seminal plasma 8-OHdG content with sperm concentration, vitality, progressive motility, and normal morphology in the varicocele group [30]. Some evidence indicated that the level of 8-OHdG in spermatozoa is negatively correlated with progressive sperm motility, normal morphology, sperm counts [15,29] and sperm concentration [29]. In agreement with the present study, Hosen et al. [28] found an inverse correlation between 8 -OHdG levels in the seminal plasma with normal morphology, sperm counts. They also found inverse correlation with sperm motility, but a lack of such association was shown in our study. The mechanisms of DNA damage in sperm that induced by the high level of 8 -oxodG is undoubtedly complex [28]. The most likely reasons for the high level of 8-oxodG in spermatozoa are OS and decreased activity of enzymes involved in DNA repair and cell death [31,32].

In our study, seminal TAC levels were significantly lower in infertile men compared to healthy fertile men (Table 2). Several investigators also have reported reductions of total antioxidant status (TAS) in seminal plasma of infertile men compared to that of fertile men $[11,18$, $28,33,34]$. The data also showed a positive correlation between sperm count, and normal morphology with TAC levels in fertile and idiopathic infertile men ( $p=0.043$ and $p=0.025$, respectively). Additionally, there was a positive and statistically insignificant correlation between the TAC and motility. In agreement with our result, some studies have shown a positive and significant correlation between sperm morphology [33] and sperm count [28] with seminal TAC levels in men with idiopathic infertility. In contrast, other studies have shown a significant association between semen TAC or TAS and sperm motility [28,33]. Also, in the study of Hosen et al. [28], there 
was no correlation between sperm count and TAC levels in either idiopathic infertile, or fertile men.

On the other hand, we found a positive association between 8-OHdG level and NO in seminal plasma while 8-OHdG was negatively correlated with TAC, but they were not statistically significant. Some studies have found a negative correlation of 8-OHdG with TAS and SOD in seminal plasma [28]. Amiri et al. [35] also reported NO levels are positively associated with 8-OHdG levels in seminal plasma of diabetic men.

Overall, these studies clearly indicated the effect of OS on the sperm oxidative DNA damage and sperm quality, hence their fertility potentials. It seems that the contradictory results achieved in the studies may be due to different types of patient populations and etiology of infertility in the subjects. However, to date, only few studies have reported correlation of NO and 8-OHdG levels in seminal plasma with male infertility. Indeed, contradictory results have been reported on the seminal plasma TAC levels between infertile and fertile males. However, several antioxidants are utilized for improvements of sperm quality, and the management of male infertility [36].

In conclusion, the present study showed higher NO and 8-OHdG levels and lower TAC levels in the seminal plasma of idiopathic infertile men than in that of healthy fertile men. Moreover, our findings demonstrate that elevated NO and 8-OHdG levels were negatively correlated with volume, total sperm counts and morphology, while TAC was positively correlated with sperm counts and morphology. Thus, it was concluded that the sperm quality is affected by the oxidative balance in semen. Increased level of NO, and 8-OHdG in seminal plasma can be considered as one of the risk factors for poor sperm quality. These results suggested that $\mathrm{OS}$ could have a negative effects on sperm function by inducing damage to the sperm DNA and decreases sperm fertilization potential. Therefore, evaluation of oxidative biomarkers may be a useful tool for male infertility diagnosis and treatment of patients with antioxidants. In addition, this study set the scene for further investigations on the possible molecular mechanisms of increased nitrate/nitrite formation, and DNA oxidative damage in seminal plasma of infertile men.

\section{Conflict of interest}

No potential conflict of interest relevant to this article was reported.

\section{Acknowledgments}

We apologize to our colleagues whose names could not be cited because of space constraints and hereby appreciate them.
ORCID

Maryam Gholinezhad https://orcid.org/0000-0002-6525-8876 Azadeh Aliarab https://orcid.org/0000-0003-0453-3958 Ghasem Abbaszadeh-Goudarzi

https://orcid.org/0000-0002-4078-6226

Yousefreza Yousefnia-Pasha https://orcid.org/0000-0001-5770-6408 Korush Rasolpour-Roshan https://orcid.org/0000-0001-9457-0730 Hemat Aghagolzadeh-Haji https://orcid.org/0000-0001-8213-6902 Milad Mohammadoo-Khorasani

https://orcid.org/0000-0002-8204-5872

\section{Author contributions}

Conceptualization: MG. Data curation: MG, AA, HAH. Formal analysis:YYP. Methodology: MG, AA, NS, KRR, HAH. Project administration: MG, GAG. Visualization: MG, KRR. Writing - original draft: MG, YYP. Writing - review \& editing: MG, GAG, MMK.

\section{References}

1. Agarwal A, Virk G, Ong C, du Plessis SS. Effect of oxidative stress on male reproduction. World J Mens Health 2014;32:1-17.

2. Bansal AK, Bilaspuri GS. Impacts of oxidative stress and antioxidants on semen functions. Vet Med Int 2010;2010:686137.

3. Layali I, Tahmasbpour E, Joulaei M, Jorsaraei SG, Farzanegi P. Total antioxidant capacity and lipid peroxidation in semen of patient with hyperviscosity. Cell J 2015;16:554-9.

4. Saleh RA, Agarwal A. Oxidative stress and male infertility: from research bench to clinical practice. J Androl 2002;23:737-52.

5. Jeng HA, Pan CH, Chao MR, Lin WY. Sperm DNA oxidative damage and DNA adducts. Mutat Res Genet Toxicol Environ Mutagen 2015;794:75-82.

6. Buzadzic B, Vucetic M, Jankovic A, Stancic A, Korac A, Korac B, et al. New insights into male (in)fertility: the importance of NO. Br J Pharmacol 2015;172:1455-67.

7. Balercia G, Moretti S, Vignini A, Magagnini M, Mantero F, Boscaro $\mathrm{M}$, et al. Role of nitric oxide concentrations on human sperm motility. J Androl 2004;25:245-9.

8. Uribe P, Boguen R, Treulen F, Sanchez R, Villegas JV. Peroxynitritemediated nitrosative stress decreases motility and mitochondrial membrane potential in human spermatozoa. Mol Hum Reprod 2015;21:237-43.

9. Du Plessis SS, McAllister DA, Luu A, Savia J, Agarwal A, Lampiao F. Effects of $\mathrm{H}(2) \mathrm{O}(2)$ exposure on human sperm motility parameters, reactive oxygen species levels and nitric oxide levels. Andrologia 2010;42:206-10. 
10. Belen Herrero $M$, Chatterjee $S$, Lefievre L, de Lamirande $E$, Gagnon C. Nitric oxide interacts with the CAMP pathway to modulate capacitation of human spermatozoa. Free Radic Biol Med 2000;29:522-36.

11. Badade G, More K, Narshetty G, Badade VZ, Yadav BK. Human seminal oxidative stress: correlation with antioxidants and sperm quality parameters. Ann Biol Res 2011;2:351-9.

12. Bianco B, Ghirelli-Filho M, Cavalheiro CM, Cavalcanti V, Peluso C, Gava MM, et al. Variants in endothelial nitric oxide synthase (eNOS) gene in idiopathic infertile Brazilian men. Gene 2013;519: 13-7.

13. Song P, Zou S, Chen T, Chen J, Wang Y, Yang J, et al. Endothelial nitric oxide synthase (eNOS) T-786C, 4a4b, and G894T polymorphisms and male infertility: study for idiopathic asthenozoospermia and meta-analysis. Biol Reprod 2015;92:38.

14. Khosravi F, Valojerdi MR, Amanlou M, Karimian L, Abolhassani F. Relationship of seminal reactive nitrogen and oxygen species and total antioxidant capacity with sperm DNA fragmentation in infertile couples with normal and abnormal sperm parameters. Andrologia 2014;46:17-23.

15. Cambi M, Tamburrino L, Marchiani S, Olivito B, Azzari C, Forti G, et al. Development of a specific method to evaluate 8-hydroxy, 2-deoxyguanosine in sperm nuclei: relationship with semen quality in a cohort of 94 subjects. Reproduction 2013;145:22735.

16. World Health Organization. Laboratory manual of the WHO for the examination of human semen and sperm-cervical mucus interaction. Ann Ist Super Sanita 2001;37:I-XII,1-123.

17. Kruger TF, Menkveld R, Stander FS, Lombard CJ, Van der Merwe JP, van Zyl JA, et al. Sperm morphologic features as a prognostic factor in in vitro fertilization. Fertil Steril 1986;46:1118-23.

18. Yousefniapasha Y, Jorsaraei G, Gholinezhadchari M, Mahjoub S, Hajiahmadi M, Farsi M. Nitric oxide levels and total antioxidant capacity in the seminal plasma of infertile smoking men. Cell J 2015;17:129-36.

19. Benzie IF, Strain JJ. The ferric reducing ability of plasma (FRAP) as a measure of "antioxidant power": the FRAP assay. Anal Biochem 1996;239:70-6.

20. Amiri I, Sheikh N, Najafi R. Nitric oxide level in seminal plasma and its relation with sperm DNA damages. Iran Biomed J 2007; 11:259-64.

21. Aksoy Y, Ozbey I, Aksoy H, Polat O, Akcay F. Seminal plasma nitric oxide concentration in oligo- and/or asthenozoospermic subjects with/without varicocele. Arch Androl 2002;48:181-5.

22. Huang I, Jones J, Khorram O. Human seminal plasma nitric oxide: correlation with sperm morphology and testosterone. Med Sci Monit 2006;12:CR103-6.
23. Revelli A, Bergandi L, Massobrio M, Lindblom B, Bosia A, Ghigo D. The concentration of nitrite in seminal plasma does not correlate with sperm concentration, sperm motility, leukocytospermia, or sperm culture. Fertil Steril 2001;76:496-500.

24. Herrero MB, de Lamirande E, Gagnon C. Tyrosine nitration in human spermatozoa: a physiological function of peroxynitrite, the reaction product of nitric oxide and superoxide. Mol Hum Reprod 2001;7:913-21.

25. Hibbs JB Jr, Vavrin Z, Taintor RR. L-arginine is required for expression of the activated macrophage effector mechanism causing selective metabolic inhibition in target cells. J Immunol 1987; 138:550-65.

26. Pacher P, Beckman JS, Liaudet L. Nitric oxide and peroxynitrite in health and disease. Physiol Rev 2007;87:315-424.

27. Sheikh N, Amiri I, Najafi R, Goodarzi MT. The correlation between total antioxidant capacity and nitric oxide concentration in seminal plasma with sperm DNA damage. Afr J Biotechnol 2010;9: 5739-45.

28. Hosen MB, Islam MR, Begum F, Kabir Y, Howlader MZ. Oxidative stress induced sperm DNA damage, a possible reason for male infertility. Iran J Reprod Med 2015;13:525-32.

29. Guz J, Gackowski D, Foksinski M, Rozalski R, Zarakowska E, Siomek $A$, et al. Comparison of oxidative stress/DNA damage in semen and blood of fertile and infertile men. PLoS One 2013;8: e68490.

30. Kao SH, Chao HT, Chen HW, Hwang TI, Liao TL, Wei YH. Increase of oxidative stress in human sperm with lower motility. Fertil Steril 2008;89:1183-90.

31. Aitken RJ, Koppers AJ. Apoptosis and DNA damage in human spermatozoa. Asian J Androl 2011;13:36-42.

32. Mishra S, Kranthi V, Kumar R, Malhotra N, Mohanty K, Pathak V, et al. Oxidative damage to sperm DNA: clinical implications. Andrology 2014;3:10000116.

33. Fazeli F, Salimi S. Correlation of seminal plasma total antioxidant capacity and malondialdehyde levels with sperm parameters in men with idiopathic infertility. Avicenna J Med Biochem 2016;4: e29736.

34. Roychoudhury S, Sharma R, Sikka S, Agarwal A. Diagnostic application of total antioxidant capacity in seminal plasma to assess oxidative stress in male factor infertility. J Assist Reprod Genet 2016;33:627-35

35. Amiri I, Karimi J, Piri H, Goodarzi MT, Tavilani H, Khodadadi I, et al. Association between nitric oxide and 8-hydroxydeoxyguanosine levels in semen of diabetic men. Syst Biol Reprod Med 2011;57: 292-5.

36. Agarwal A, Majzoub A. Role of antioxidants in assisted reproductive techniques. World J Mens Health 2017;35:77-93. 